\title{
Suicidal gene therapy of leiomyoma via delivery of herpes thymidine kinase gene by means of $\alpha v \beta 3$ integrin-targeted peptide-based carriers and magnetic nanoparticles.
}

\section{S.V. Shtykalova ${ }^{1,2, *}$, A.A. Egorova ${ }^{1}$, A.V. Selyutin ${ }^{1}$, M.A. Maretina ${ }^{1}$, S.A. Selkov ${ }^{1}$, V.S. Baranov ${ }^{1,2}$, A.V. Kiselev ${ }^{1 *}$}

1 - Institute of Obstetrics, Gynecology and Reproductology named after D.O. Ott, Saint-Petersburg, Russia

2 - Department of Genetics \& Biotechnology, Saint Petersburg State University, Saint-Petersburg, Russia

*Corresponding authors, e-mails: sofia.shtykalova@gmail.com; ankiselev@yahoo.co.uk

\section{Introduction}

Uterine leiomyoma $(\mathrm{UL})$ is the most common benign tumor of the female reproductive tract. Precise ultrasound localization of tumors and their availability by means of different endoscopic techniques makes UL a perfect target for suicidal gene therapy in situ using e.g. herpes thymidine kinase (HSV1-TK) gene transfer. Development of specific and efficient carriers for targeted delivery of genetic constructs to cells is a current problem of gene therapy. We developed novel RGD-targeted peptide-based carriers for plasmid DNA delivery into the av $\beta 3$-expressing cells, including primary UL cells. For successful penetration into the inner layers of the UL, magnetic nanoparticles (MNPs) were non-covalently bound to DNA-peptide complexes.

\section{Materials and methods}

The efficiency of DNA condensation in the polyplexes with MNPs was evaluated by the quenching of ethidium bromide. DNA stability in the polyplexes with MNPs was studied using a DNAase I protection assay. The specificity of the cell penetration and DNA delivery have been demonstrated by ligand-competitive DNA uptake and transfection experiments performed on PANC-1 cells. Suicidal gene therapy with HSV1TK gene encoding plasmid PPTK-1 and subsequent ganciclovir treatment was held for primary leiomyoma cells at early passages.

\section{Results}

Non-viral carriers composed of RGD ligand-conjugated arginine-rich peptides and ligand-free peptides were synthesized. Physicochemical properties of DNA-complexes with MNPs have been studied.

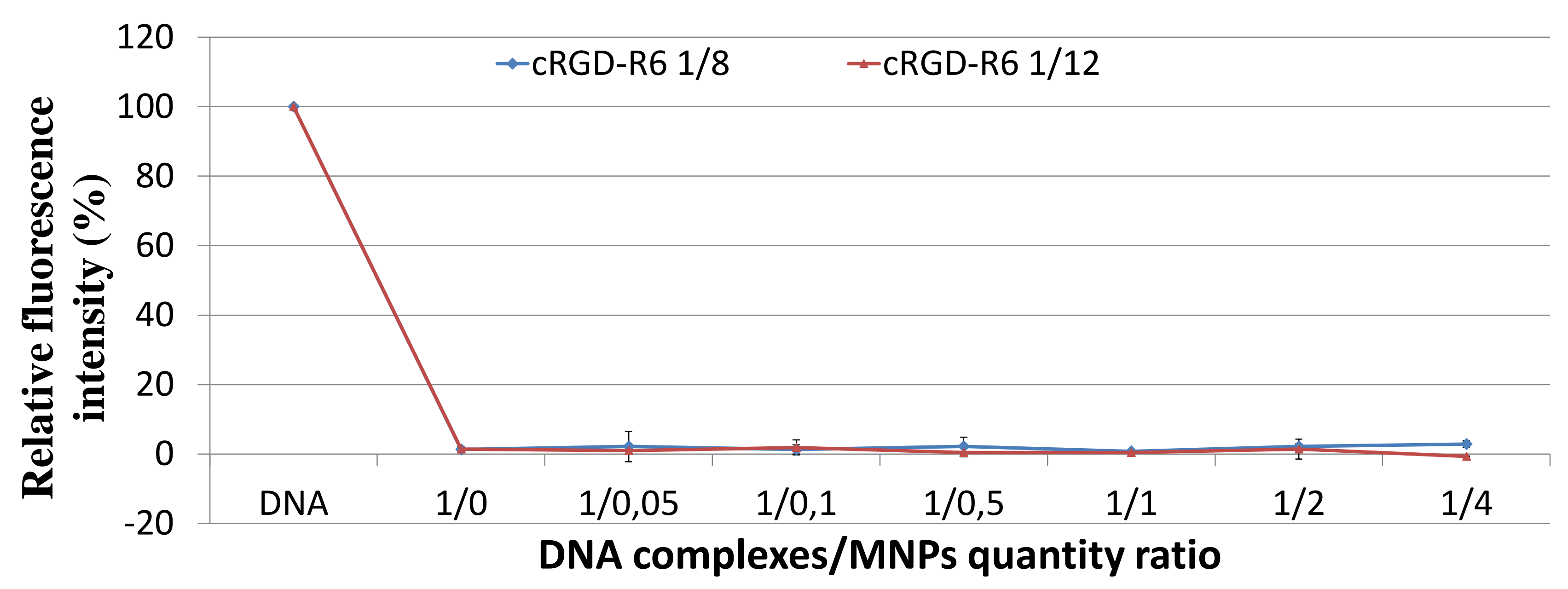

Fig. 1 - Study of DNA/cRGD-R6 complexes stability with increasing weight ratios of DNAcomplexes and magnetic nanoparticles. The results are presented as mean and S.D.

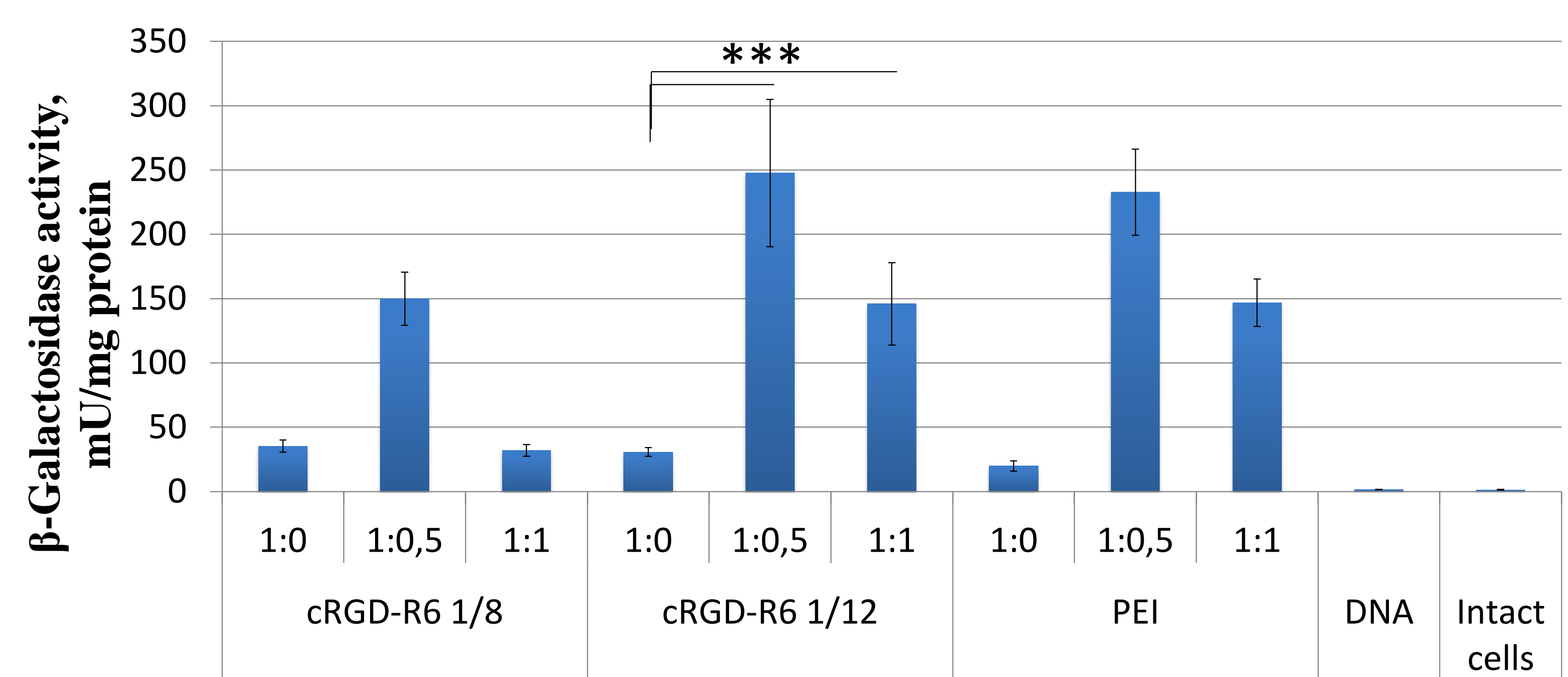

Fig. 2 - Activity of $\beta$-galactosidase in PANC-1 cells after magnetofection with pCMV-LacZ complexes with CRGD-R6 carrier and MNPs. The results are presented as mean and S.E.M. $(* * *$ - p-value $<0.005)$.

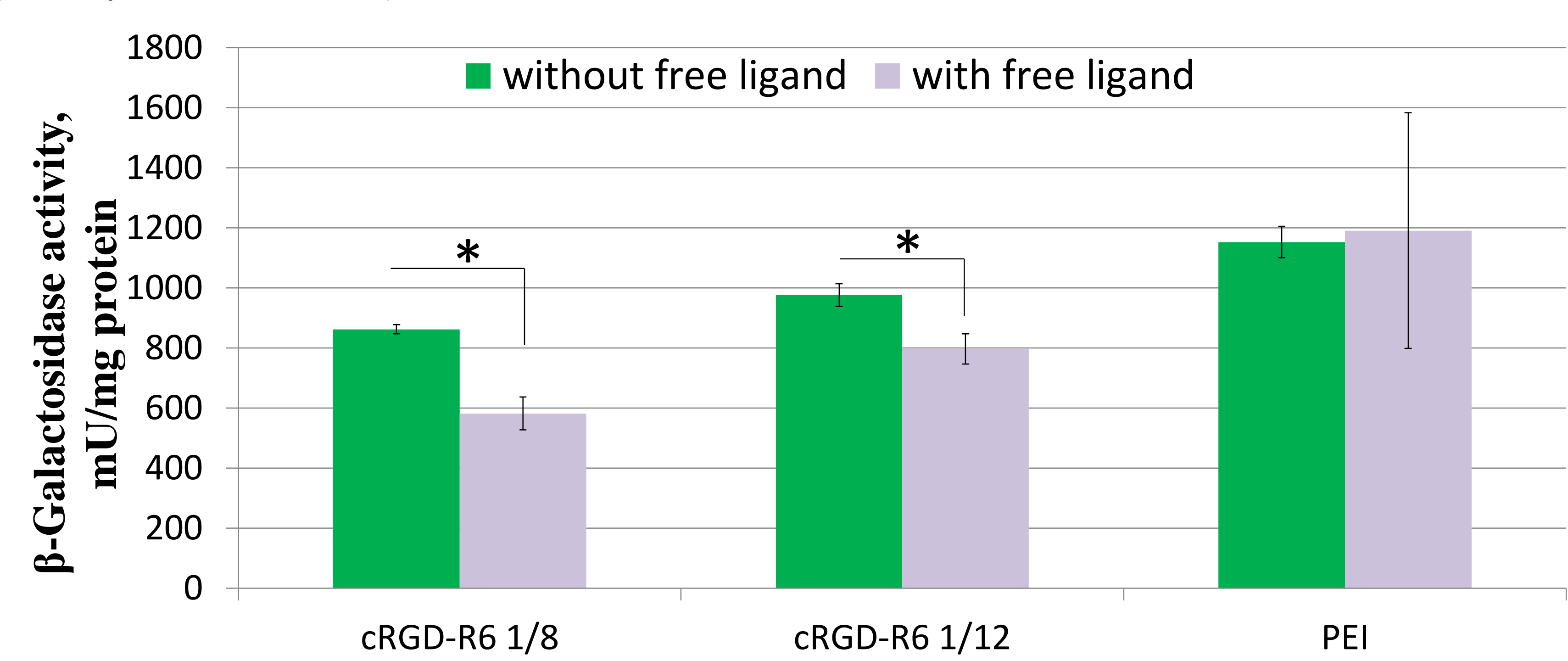

Fig. 3 - Activity of $\beta$-galactosidase after magnetofection of PANC-1 cells with nucleopeptide complexes with MNPs with and without the addition of a free ligand $*^{*}$ p.value $<0.05$ ).

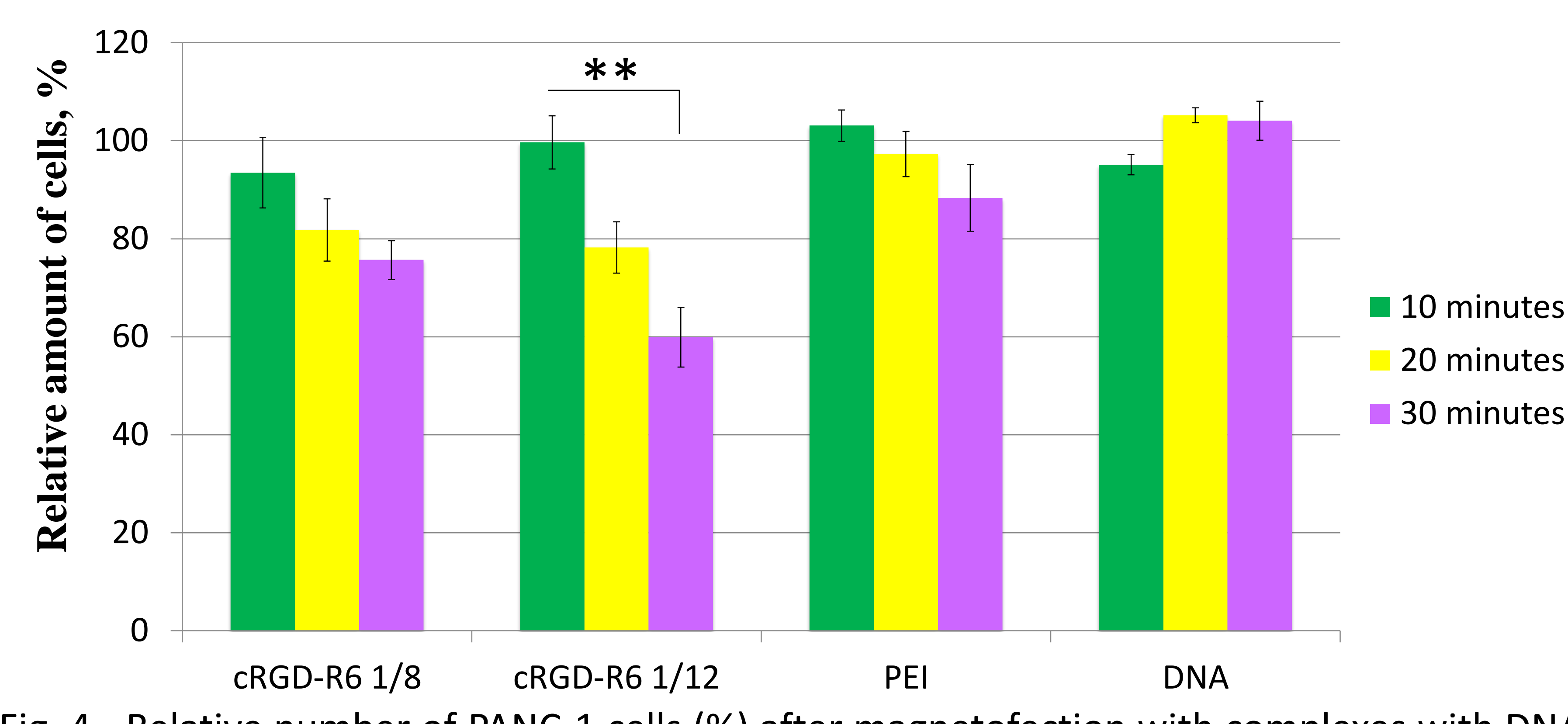

Fig. 4 - Relative number of PANC-1 cells (\%) after magnetofection with complexes with DNA / CRGD-R6 and MNPs. DNA:MNP weight ratio is 1:0.5 and different incubation times of complexes with cells $(10,20$, and 30 minutes) $p$-value $<0.01)$.

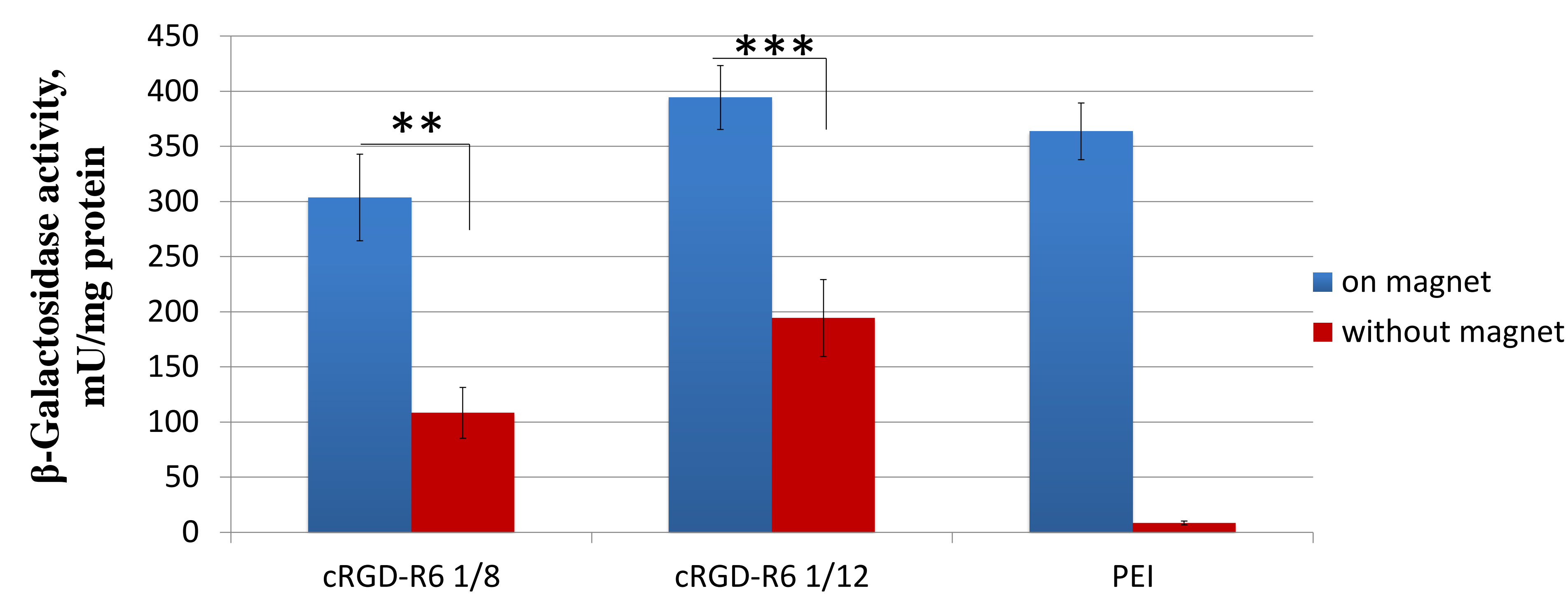

Fig. 5 - Activity of $\beta$-galactosidase after transfection of PANC-1 cells with nucleopeptide complexes with MNPs in the presence and absence of a magnet $(* *-$ p.value $<0,01, * * *$ p.value < 0.005).

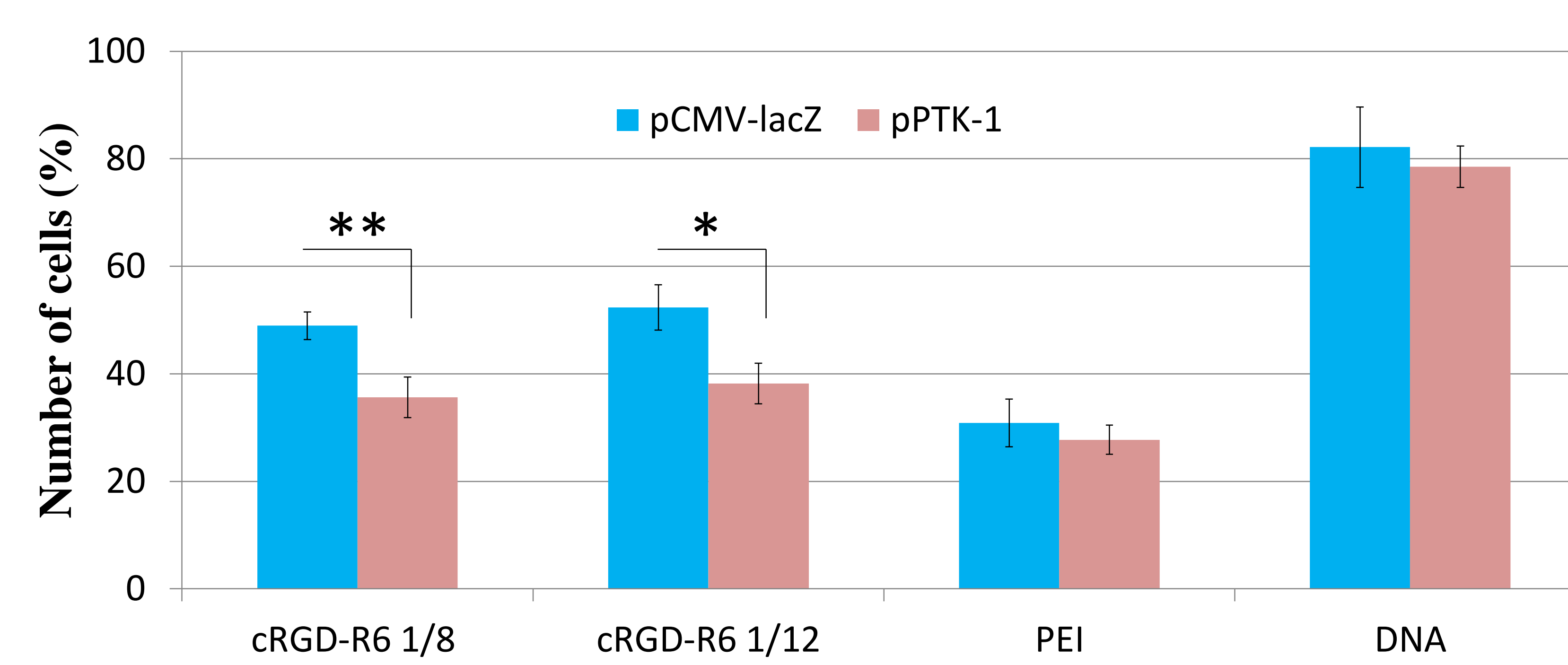

Fig. 6 - Results of Alamar blue assay after transfection of primary uterine leiomyoma cells after transfection with DNA-complexes with MNPs (DNA:MNP weight ratio is $1: 0.5)\left({ }^{*}-p\right.$-value < $0.05, * *$ - p-value $<0.01)$.
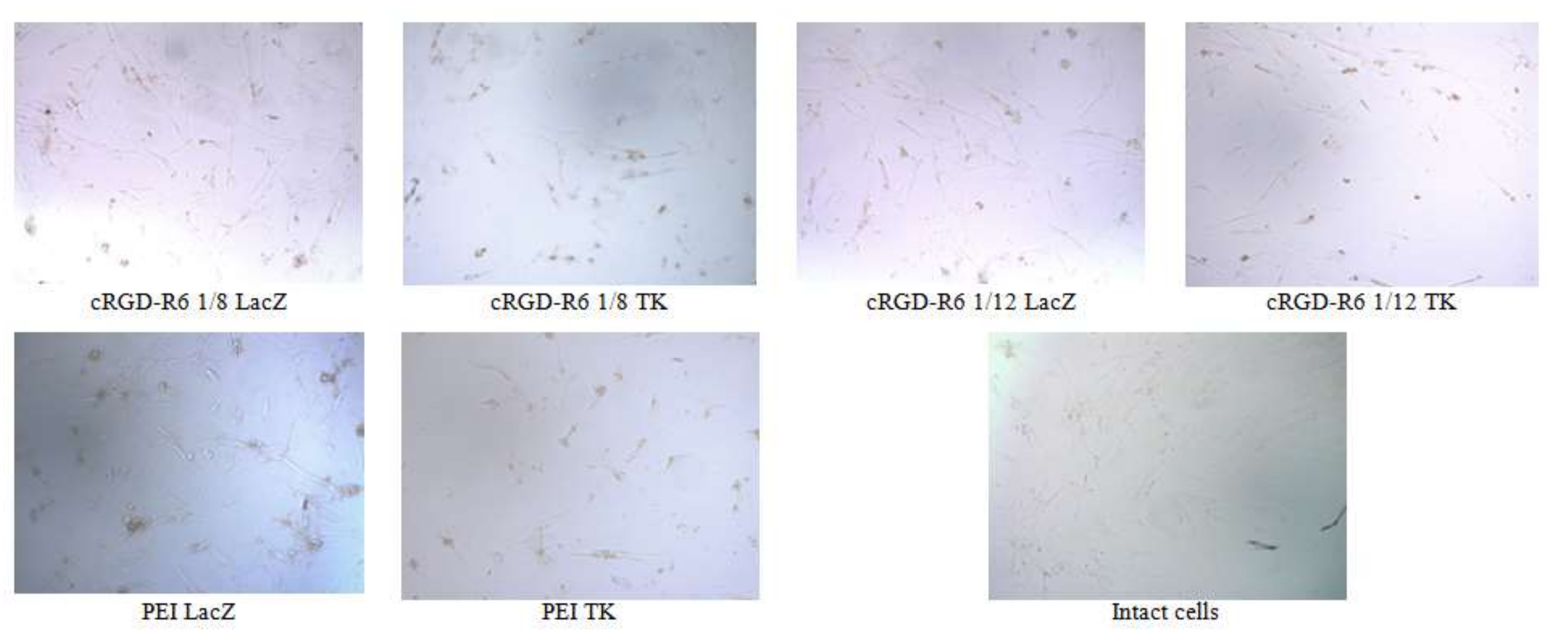

Fig. 7 - Uterine leiomyoma cells after magnetofection with DNA-peptide polyplexes with MNPs (DNA:MNP weight ratio 1:0.5) carrying the LacZ gene and with the HSV1-TK gene.

\section{Conclusion}

The developed DNA-complexes with MNP demonstrated high specificity and transfection efficiency of leiomyoma cells with subsequent successful suicide gene therapy, which makes them promising for the development of UL gene therapy.

\section{Acknowledgements}

The research is supported by RSF grant 19-15-00108. Marianna Maretina is supported by a President of Russian Federation personal scholarship (SP822.2018.4). 